\title{
Neurofobia em uma escola médica privada: prevalência e consequências no processo ensino-aprendizagem
}

\author{
Neurophobia in a private medical school: prevalence and consequences in the teaching- \\ learning process
}

Neurofobia en una escuela de medicina privada: prevalencia y consecuencias en el processo de enseñanza-aprendizaje

Artur dos Santos Soares ${ }^{1 *}$, Adenilso Pinheiro da Silva ${ }^{1}$, Amanda Santos Duarte ${ }^{1}$, Ana Bárbara Barbosa Roque ${ }^{1}$, Joel Ferreira de Jesus Neto ${ }^{1}$, Celina Cláudia Israel Sefer ${ }^{1}$.

\section{RESUMO}

Objetivo: Avaliar a percepção e o grau dificuldade dos estudantes de Medicina em relação ao processo ensino aprendizagem em Neurologia voltada para prática médica. Métodos: Estudo transversal, analítico o e quantitativo, no qual serão aplicados questionários com perguntas diretas e respostas objetivas aos acadêmicos de medicina do $4^{\circ}$ ao $8^{\circ}$ semestre do curso de Medicina de uma instituição do Pará, no período de setembro a dezembro de 2019. Sendo a amostragem probabilística 161 alunos com coleta de dados em agosto de 2020, na qual será usado um questionário próprio autoaplicável, onde serão abordadas perguntas pertinentes referentes ao nível de interesse, como os estudantes de Medicina se sentem em relação ao processo de ensino e aprendizagem, o grau de confiança para resolução de emergências Neurológicas e fatores que interferem no processo de ensino-aprendizagem dos estudantes de Medicina. Resultados: $O$ sexo feminino foi o de maior incidência significante $\left({ }^{*} p=0.0016\right)$ entre os discentes entrevistados $(62.7 \%)$. $\mathrm{Na}$ comparação entre os períodos analisados, não houve diferença significante $(p=0.7014)$ na proporção entre os gêneros de acordo com os períodos do curso Conclusão: Há existência da neurofobia de acordo com a preparação e metodologia de ensino da especialidade.

Palavras-chave: Neurologia, Ensino, Deficiências de aprendizagem.

\begin{abstract}
Objective: To evaluate the perception and the degree of difficulty of medical students in relation to the teaching-learning process in Neurology aimed at medical practice. Methods: Cross-sectional, analytical and quantitative study, in which questionnaires with direct questions and objective answers will be applied to medical students from the 4th to the 8th semester of the Medicine course at an institution in Pará, from September to December 2019. probabilistic sampling 161 students with data collection in August 2020, in which a self-administered questionnaire will be used, which will address pertinent questions regarding the level of interest, how medical students feel about the teaching process and learning, the degree of confidence to resolve Neurological emergencies and factors that interfere in the teaching-learning process of medical students. Results: The female sex was the one with the highest significant incidence ( $\left.{ }^{*} p=0.0016\right)$ among the interviewed students (62.7\%). In the comparison between the periods analyzed, there was no significant difference $(p=0.7014)$ in the proportion between genders according to the periods of the course. Conclusion: There is the existence of neurophobia according to the preparation and teaching methodology of the specialty.
\end{abstract}

Keywords: Neurology, Teaching, Learning disabilities.

${ }^{1}$ Centro Universitário Metropolitano da Amazônia (UNIFAMAZ), Belém - PA.

*E-mail: artur_soares_@hotmail.com 


\section{RESUMEN}

Objetivo: Evaluar la percepción y el grado de dificultad de los estudiantes de medicina en relación al proceso de enseñanza-aprendizaje en Neurología dirigido a la práctica médica. Métodos: Estudio transversal, analítico y cuantitativo, en el que se aplicarán cuestionarios con preguntas directas y respuestas objetivas a estudiantes de medicina del 40 al 80 semestre de la carrera de Medicina en una institución de Pará, de septiembre a diciembre de 2019. muestreo probabilístico 161 estudiantes con recopilación de dados en agosto de 2020, en el cual se utilizará un cuestionario autoadministrado, que abordará preguntas pertinentes sobre el nivel de interés, cómo se sienten los estudiantes de medicina sobre el proceso de enseñanza y aprendizaje, el grado de confianza para resolver emergencias neurológicas y factores que interfieren en el proceso de enseñanzaaprendizaje de los estudiantes de medicina. Resultados: El sexo femenino fue el de mayor incidencia significativa ( $\left.{ }^{*} p=0,0016\right)$ entre los estudiantes entrevistados $(62,7 \%)$. En la comparación entre los periodos analizados, no hubo diferencia significativa $(p=0,7014)$ en la proporción entre géneros según los periodos de la asignatura. Conclusión: Existe existencia de neurofobia según la metodología de preparación y enseñanza de la especialidad.

Palabras clave: Neurología, Enseñanza, Discapacidades para el aprendizaje.

\section{INTRODUÇÃO}

Uma das especialidades mais frequentes durante o atendimento ambulatorial ou urgência e emergência são sinais, sintomas e patologias da área neurológica. No Brasil, entre 5-20\%, dependendo da localidade e da região, são atendimentos de doenças da Neurologia. Além disso, segundo dados da Organização Mundial da Saúde (OMS), patologias da área neurológica somam até 6,3\% da carga total para a saúde e possuem impacto na saúde pública, pois muitas delas são relacionadas ao processo de envelhecimento populacional tais como Doença de Alzheimer e outras patologias neurodegenerativas.

Bem como doenças relacionadas a envelhecimento, diversas patologias comumente encontradas também fazem parte da seara da neurologia tais como acometimento neurológico em doenças como hanseníase, consequências crônicas do diabetes mellitus, acidente vascular encefálico e cefaleia/enxaqueca e, por serem comuns, deveriam receber mais atenção pelos discentes de medicina durante o curso (GELB DJ, 2002).

Contudo, isso não ocorre. Muitos estudantes de medicina englobam a neurologia e generalizam-na em estudos anatômicos, fisiológicos, bioquímicos complexos que acabam por bloquear o processo ensinoaprendizagem desta especialidade durante o curso e impedem a construção de habilidades e competências para o futuro médico sendo esse processo chamado de "Neurofobia" (MATTHIAS AT, et al., 2013).

Essa aversão é iniciada, em diversos pontos durante a graduação: no início do curso com relatos de estudantes veteranos expressando dificuldades durante o curso repassam e constroem 0 medo em estudantes calouros, durante o estudo da especialidade ao iniciar com estudos anatômicos e fisiológicos complexos sem contextualização ou posterior ao período de estudo do módulo quando o discente conclui um balanço negativo de aprendizado e gera bloqueios para futuras revisitações de conteúdo (JOZEFOWICZ RF, et al., 1994; ALBERT DA, et al., 2015).

O estudante desenvolvendo aversão pela neurologia e bloqueando os conhecimentos que podem vir a encontrar durante o curso pode diminuir a possibilidade de conseguir diagnosticar patologias que poderiam ser resolvidas na atenção básica ou pelo clínico médico, encaminhando muitas vezes o paciente para neurologistas, entrando em uma fila de espera que pode demorar anos para uma consulta (ALBERT DA, et al., 2015).

No Brasil, há uma desigualdade na concentração de neurologistas, sendo uma grande parte residindo na região Sul e Sudeste do país. Estudos de 2014 demonstram que a região Norte possui média de 0,68 neurologistas por 100.000 habitantes (PRITHISHKUMAR IJ e HOLLA SJ, 2012; BRASIL, 2015). 
Dessa forma, com um número pequeno de neurologistas por habitante demonstrando a pequena quantidade no país, ocorrem taxas altas de subnotificação de doenças ou diagnósticos tardios pela quantidade de pessoas em lista de espera para consultas com essa especialidade fazendo demorar em média de 9 meses até 1 anos para uma consulta especializada, piorando a qualidade de vida. Sendo assim se houvesse menos neurofobia, algumas patologias neurológicas básicas poderiam ser tratadas ou acompanhadas em atenção básica e/ou clínica médica, diminuindo a quantidade de pessoas encaminhadas fazendo com que os especialistas recebessem pacientes com patologias mais específicas da área (FIESTAS F, et al., 2009).

Essa realidade é um reflexo da neurofobia durante o curso onde discente ao não desenvolver as habilidades básicas requeridas pelo estudo ao passar pelo conteúdo da área neurológica, além de se sentir despreparado para atender patologias ambulatoriais e em urgência e emergência básicas dessa área, diminui a possibilidade de atendimento eficiente para os pacientes que necessitam, aumentando filas de espera, superlotando consultórios médicos especializados, aumentando o tempo entre diagnóstico e manejo de doenças que deveriam fazer parte do conhecimento do médico generalista (FIESTAS F, et al., 2009).

Com isso, este estudo teve como objetivo avaliar a percepção e o grau dificuldade dos estudantes de Medicina em relação ao processo ensino aprendizagem em Neurologia voltada para prática médica.

\section{MÉTODOS}

O presente projeto é baseado no estudo de Schon e col, com o tema "Is clinical neurology really so difficult?', publicado na revista Journal of Neurology, Neurosurgery \& Psychiatry, no ano de 2002. Este estudo é transversal, analítico e quantitativo, no qual serão aplicados questionários com perguntas diretas e respostas objetivas aos acadêmicos de medicina do $4^{\circ}$ ao $8^{\circ}$ semestre do curso de Medicina de uma instituição de ensino superior do Norte do Brasil.

Foram incluídos na pesquisa todos os alunos que concordarem em participar da mesma, após esclarecimentos dos seus objetivos, métodos, riscos e benefícios, e que estejam regularmente matriculados no curso de Medicina do 4ำ ao $8^{\circ}$ semestre. Foram excluídos desta pesquisa os acadêmicos que responderem o questionário de forma incompleta, que desistirem do curso ao longo da pesquisa e os que não completarem todas as etapas das avaliações necessárias.

Serão excluídas do estudo as turmas do $1^{\circ}$ e $3^{\circ}$ semestre, em virtude de ainda não terem tido contado com conteúdo de Neurologia e Neurociências, e também as turmas do internato, devido às essas estarem inseridos ao ciclo clínico-pratico, e este trabalho visa avaliar apenas os estudantes que estão no ciclo clinico.

Sendo a amostragem probabilística 161 alunos com coleta de dados em agosto de 2020, na qual foi usado um questionário próprio autoaplicável, onde foram abordadas perguntas pertinentes referentes o nível de interesse, como os estudantes de Medicina se sentem em relação ao processo de ensino e aprendizagem, o grau de confiança para resolução de emergências Neurológicas e fatores que interferem no processo de ensino-aprendizagem dos estudantes de Medicina.

Para análise dos dados foram calculados média e respectivo desvio padrão bem como a frequência relativa e absoluta dependendo da natureza da variável utilizada. Os dados coletados foram inseridos numa planilha do software Microsoft Excel 2010 e expostos por gráficos e/ou tabelas, as variáveis quantitativas serão expressas sob a forma de média \pm , desvio padrão e as variáveis categóricas serão expressas através de frequências absolutas e relativas no software BioEstat 5.0.

A pesquisa foi realizada após a aprovação do Comitê de Ética em Pesquisa (CEP) sob número de parecer ํo 4.188.660.

\section{RESULTADOS}

O sexo feminino foi o de maior incidência significante $\left({ }^{*} p=0.0016\right)$ entre os discentes entrevistados (62.7\%). Na comparação entre os períodos analisados, não houve diferença significante $(p=0.7014)$ na proporção entre os gêneros de acordo com os períodos do curso (Tabela 1). 
Tabela 1 - Discentes segundo o gênero, de acordo com o período do curso.

\begin{tabular}{ccccc}
\hline \multirow{2}{*}{ Período do curso } & \multicolumn{2}{c}{ Feminino* $^{2}$ Masculino } \\
\cline { 2 - 5 } & Freq & $\%$ & Freq & $\%$ \\
\hline Quarto & 36 & $35.6 \%$ & 18 & $30.0 \%$ \\
Quinto & 17 & $16.8 \%$ & 14 & $23.3 \%$ \\
Sexto & 17 & $16.8 \%$ & 11 & $18.3 \%$ \\
Sétimo & 19 & $18.8 \%$ & 8 & $13.3 \%$ \\
Oitavo & 12 & $11.9 \%$ & 9 & $15.0 \%$ \\
\hline Total & $\mathbf{1 0 1}$ & $\mathbf{6 2 . 7 \%}$ & $\mathbf{6 0}$ & $\mathbf{3 7 . 3 \%}$
\end{tabular}

Legenda: $p=0.7014$ Teste Qui-Quadrado Independência; ${ }^{*} p=0.0016$ Teste Qui-Quadrado Aderência. Fonte: SOARES AS, et al., 2020.

A idade dos discentes difere significativamente $\left({ }^{*} \mathrm{p}=0.0002\right)$ em suas faixas etárias. A faixa etária de 20 a 24 anos é a que possui maior percentual no quarto (79.6\%), no quinto ( $41.9 \%)$, no sexto $(71.4 \%)$ e no sétimo período (59.3\%). No oitavo período, a faixa de maior proporção é a de 25 a 29 anos (42.9\%).

Não houve diferença significante $(p=0.0503)$ entre os períodos estudados, em relação a ter uma graduação anterior. Ao analisar o grupo, no geral, houve proporção significante $\left({ }^{*} p<0.0001\right)$ de discentes que não possuem graduação anterior (84.5\%).

Em relação à especialidade que os discentes consideram de maior complexidade, foi encontrada diferença significante $\left({ }^{*} p=0.0018\right)$ nas proporções entre os períodos estudados. A opinião sobre a complexidade da Neurologia é maior no quarto período e diminui proporcionalmente com o passar dos semestres, chegando a $18.7 \%$ no oitavo semestre.

No geral, a especialidade considerada de maior complexidade foi à neurologia (25.2\%), seguida pela cardiologia (23.5\%). A reumatologia é considerada a de menor complexidade, por apenas $14.7 \%$ dos alunos. O nível de interesse dos discentes pela neurologia apresentou proporções significativamente diferentes $\left({ }^{*} \mathrm{p}=\right.$ $0,0369)$, mostrando um maior interesse pela disciplina no oitavo período $(52.4 \%)$ e o menor interesse no quarto período (14.8\%). No geral, o nível de interesse ficou em $64.0 \%$, variando entre moderado (37.3\%) a muito $(26.7 \%)$ (Tabela 2).

Tabela 2 - Discentes segundo o nível de interesse pela neurologia, de acordo com o período do curso.

\begin{tabular}{ccccccccc}
\hline \multirow{2}{*}{ Período } & \multicolumn{7}{c}{ Nível de interesse pela Neurologia } \\
\cline { 2 - 10 } & \multicolumn{2}{c}{ Nenhum } & \multicolumn{2}{c}{ Pouco } & \multicolumn{7}{c}{ Moderado } & Muito \\
\hline Quarto & 8 & $14.8 \%$ & 12 & $22.2 \%$ & 24 & $44.5 \%$ & 10 & $18.5 \%$ \\
Quinto & 1 & $3.2 \%$ & 7 & $22.6 \%$ & 15 & $48.4 \%$ & 8 & $25.8 \%$ \\
Sexto & 2 & $7.1 \%$ & 8 & $28.6 \%$ & 9 & $32.1 \%$ & 9 & $32.1 \%$ \\
Sétimo & 2 & $7.4 \%$ & 10 & $37.0 \%$ & 10 & $37.0 \%$ & 5 & $18.5 \%$ \\
Oitavo & 0 & $0.0 \%$ & 8 & $38.1 \%$ & 2 & $9.5 \%$ & 11 & $52.4 \%$ \\
\hline Total & 13 & $8.0 \%$ & 45 & $28.0 \%$ & 60 & $37.3 \%$ & 43 & $26.7 \%$ \\
\hline
\end{tabular}

Legenda: ${ }^{*} p=0.0369$ Teste Qui-Quadrado Partição.

Fonte: SOARES AS, et al., 2020.

O atual nível de conhecimento sobre neurologia, segundo a opinião dos discentes, variou apenas entre pouco e moderado, apresentando diferença significante $\left({ }^{*} p=0.0464\right)$ entre os períodos, sendo o oitavo ano a apresentar maior proporção de conhecimento moderado (81.0\%) e o sexto ano, o menor (60.7\%) (Tabela 3). 
Tabela 3 - Discentes segundo o nível atual de conhecimento sobre neurologia, de acordo com o período do curso.

\begin{tabular}{ccccc}
\hline \multirow{2}{*}{ Período } & \multicolumn{4}{c}{ Nível atual de conhecimento sobre Neurologia } \\
\cline { 2 - 5 } & 16 & $29.6 \%$ & 38 & \multicolumn{2}{c}{ Moderado } \\
\hline Quarto & 7 & $22.6 \%$ & 24 & $70.4 \%$ \\
Quinto & 11 & $39.3 \%$ & 17 & $77.4 \%$ \\
Sexto & 6 & $22.2 \%$ & 21 & $60.7 \%$ \\
Sétimo & 4 & $19.0 \%$ & 17 & $77.8 \%$ \\
Oitavo & 44 & $27.3 \%$ & 117 & $81.0 \%$ \\
\hline Total & &
\end{tabular}

Legenda: ${ }^{*} p=0.0464$ Teste Qui-Quadrado Independência.

Fonte: SOARES AS, et al., 2020.

Baseados em suas experiências, os discentes classificaram o processo ensino aprendizagem em neurologia de difícil a muito difícil em $93.9 \%$ dos casos. Apenas $3.1 \%$ considerou este processo fácil, havendo diferença significante ( ${ }^{*} p=0.0123$ ) entre os períodos, onde o oitavo período apresentou maior proporção na classificação "Moderado" (61.9\%) e o sétimo período na classificação "Fácil" (14.8\%) (Tabela 4).

Tabela 4 - Discentes segundo a classificação do processo de ensino aprendizagem em neurologia, segundo suas experiências, de acordo com o período do curso.

\begin{tabular}{ccccccccc}
\hline \multirow{2}{*}{ Período } & \multicolumn{6}{c}{ Baseado em sua experiência como você classificaria o processo de ensino } \\
\cline { 2 - 8 } & \multicolumn{2}{c}{ Fácil } & \multicolumn{5}{c}{ Difícil } & \multicolumn{3}{c}{ Moderado } & Muito difícil \\
\hline Quarto & 1 & $1.9 \%$ & 22 & $40.7 \%$ & 21 & $38.9 \%$ & 10 & $18.5 \%$ \\
Quinto & 0 & $0.0 \%$ & 15 & $48.4 \%$ & 14 & $45.2 \%$ & 2 & $6.5 \%$ \\
Sexto & 0 & $0.0 \%$ & 12 & $42.9 \%$ & 13 & $46.4 \%$ & 3 & $10.7 \%$ \\
Sétimo & 4 & $14.8 \%$ & 5 & $18.5 \%$ & 16 & $59.3 \%$ & 2 & $7.4 \%$ \\
Oitavo & 0 & $0.0 \%$ & 7 & $33.3 \%$ & 13 & $61.9 \%$ & 1 & $4.8 \%$ \\
\hline Total & 5 & $3.1 \%$ & 61 & $37.9 \%$ & 77 & $47.8 \%$ & 18 & $11.2 \%$ \\
\hline
\end{tabular}

Legenda: * $p=0.0123$ Teste Qui-Quadrado Partição.

Fonte: SOARES AS, et al., 2020.

$\mathrm{Na}$ análise do nível de confiança para atendimento de paciente com quadro de emergência neurológica, houve proporção significante $\left({ }^{*} p<0.0001\right)$ de discentes que declararam "Pouco" $(50.3 \%)$. As proporções mudam significativamente $\left({ }^{*} p=0.0034\right)$, de acordo com os períodos, sendo o quarto período a apresentar maior proporção de pouca confiança $(61.1 \%)$ e o oitavo período a apresentar maior proporção de moderada confiança (61.9\%).

Em relação ao atendimento de um paciente ambulatorial com quadro de doença neurológica, houve proporção significante ( $\left.{ }^{*} p<0.0001\right)$ de discentes que declararam nível "Moderado" $(54.7 \%)$ de confiança. As proporções mudam significativamente $\left({ }^{*} p=0.0377\right)$, de acordo com os períodos, sendo o quarto período a apresentar maior proporção de pouca confiança (7.4\%) e o sexto período a apresentar maior proporção de muita confiança $(28.6 \%)$.

A classificação do conhecimento básico em neurociência variou significativamente ( $\left.{ }^{*} \mathrm{p}=0.0238\right)$ entre os períodos estudados. O sétimo período apresentou maior proporção $(51.9 \%)$ de nível "Baixo" e, juntamente com o quinto e o oitavo períodos, nenhum discente $(0.0 \%)$ declarou "Alto" conhecimento.

No geral, houve proporção significante (" $p$ < 0.0001) de discentes que declararam "Moderado" conhecimento básico em neurociência, dentre os pesquisados (Tabela 5). 
Tabela 5 - Discentes segundo o conhecimento básico em neurociência, de acordo com o período do curso.

\begin{tabular}{ccccccccc}
\hline \multirow{2}{*}{ Período } & \multicolumn{6}{c}{ Como classificaria o seu conhecimento básico em Neurociência? } \\
\cline { 2 - 8 } & \multicolumn{3}{c}{ Nenhum } & \multicolumn{3}{c}{ Baixo } & \multicolumn{3}{c}{ Moderado ${ }^{\star *}$} & \multicolumn{1}{c}{ Alto } \\
\hline Quarto & 0 & $0.0 \%$ & 16 & $29.6 \%$ & 36 & $66.7 \%$ & 2 & $3.7 \%$ \\
Quinto & 1 & $3.2 \%$ & 12 & $38.7 \%$ & 18 & $58.1 \%$ & 0 & $0.0 \%$ \\
Sexto & 0 & $0.0 \%$ & 12 & $42.9 \%$ & 15 & $53.6 \%$ & 1 & $3.6 \%$ \\
Sétimo & 1 & $3.7 \%$ & 14 & $51.9 \%$ & 12 & $44.4 \%$ & 0 & $0.0 \%$ \\
Oitavo & 0 & $0.0 \%$ & 7 & $33.3 \%$ & 14 & $66.7 \%$ & 0 & $0.0 \%$ \\
\hline Total & 2 & $1.2 \%$ & 61 & $37.9 \%$ & 95 & $59.0 \%$ & 3 & $1.9 \%$ \\
\hline
\end{tabular}

Legenda: ${ }^{*} p=0.0238$ Teste Qui-Quadrado Partição; ** $p<0.0001$ Teste Qui-Quadrado Aderência.

Fonte: SOARES AS, et al., 2020.

A classificação da complexidade do exame clínico neurológico obteve uma proporção significativa ( ${ }^{*} p<$ $0.0001)$ de "Moderado" (55.9\%).

Também foi observada uma diferença significante $\left({ }^{*} p=0.0238\right)$ na classificação de acordo com os períodos estudados, sendo o oitavo período a obter a maior proporção de classificação "Muito" (14.3\%) e o sétimo período da classificação "Nenhum" (29.6\%).

A opinião dos discentes em relação à dificuldade de aprendizagem em neurologia devido a complexidade no domínio da neuroanatomia variou significativamente $\left({ }^{*} p=0.0014\right)$ entre os períodos estudados. O sexto período obteve maiores proporções entre o "pouco" (50.0\%) e o "Moderado" (46.4\%). Os períodos que consideraram de "Moderado" a "Muito" complexo, foram o quinto (45.2\%) e oitavo (42.9\%) respectivamente.

No geral houve uma proporção significante $\left({ }^{*} p<0.0001\right)$ de discentes que avaliaram como "Moderado" (50.3\%) em relação as demais classificações.

A opinião dos discentes em relação à dificuldade de aprendizagem em neurologia devido a mesma ter reputação de ser difícil apresentou diferença significativa $\left({ }^{*} p=0.0007\right)$ entre os períodos. O sexto período foi o que obteve a maior proporção de "Muito" (82.1\%) e o sétimo a maior proporção de "Nenhum" $(22.2 \%)$ (Tabela 6).

Tabela 6 - Dificuldade de aprendizagem em neurologia devido ter reputação de ser difícil, de acordo com o período do curso.

\begin{tabular}{ccccccccc}
\hline \multirow{2}{*}{ Período } & \multicolumn{7}{c}{ Neurologia ter reputação de ser difícil } \\
\cline { 2 - 9 } & \multicolumn{2}{c}{ Nenhum } & \multicolumn{7}{c}{ Pouco } & \multicolumn{1}{c}{ Moderado } & Muito \\
\hline Quarto & 2 & $3.7 \%$ & 14 & $25.9 \%$ & 13 & $24.1 \%$ & 25 & $46.3 \%$ \\
Quinto & 2 & $6.5 \%$ & 5 & $16.1 \%$ & 12 & $38.7 \%$ & 12 & $38.7 \%$ \\
Sexto & 0 & $0.0 \%$ & 1 & $3.6 \%$ & 4 & $14.3 \%$ & 23 & $82.1 \%$ \\
Sétimo & 6 & $22.2 \%$ & 7 & $25.9 \%$ & 8 & $29.6 \%$ & 6 & $22.2 \%$ \\
Oitavo & 1 & $4.8 \%$ & 4 & $19.0 \%$ & 8 & $38.1 \%$ & 8 & $38.1 \%$ \\
\hline Total & 11 & $6.8 \%$ & 31 & $19.3 \%$ & 45 & $28.0 \%$ & 74 & $46.0 \%$ \\
\hline
\end{tabular}

Legenda: * $p=0.0007$ Teste Qui-Quadrado Partição.

Fonte: SOARES AS, et al., 2020.

A dificuldade de aprendizagem em neurologia devido a mesma ter um amplo número de diagnóstico, apresentou diferença significativa ( ${ }^{*} p=0.0207$ ) entre os períodos. O sétimo período foi o único que obteve proporção $(7.4 \%)$ na categoria "Nenhum", mesmo tendo apresentado o maior valor na opção "Moderado" (55.6\%) (Tabela 7$)$. 
Tabela 7 - Dificuldade de aprendizagem em neurologia devido ter um amplo número de diagnóstico, de acordo com o período do curso.

\begin{tabular}{ccccccccc}
\hline \multirow{2}{*}{ Período } & \multicolumn{6}{c}{ Neurologia tem um amplo número de diagnóstico } \\
\cline { 2 - 9 } & \multicolumn{2}{c}{ Nenhum } & \multicolumn{2}{c}{ Pouco } & \multicolumn{2}{c}{ Moderado } & \multicolumn{2}{c}{ Muito } \\
\hline Quarto & 0 & $0.0 \%$ & 8 & $14.8 \%$ & 26 & $48.1 \%$ & 20 & $37.0 \%$ \\
Quinto & 0 & $0.0 \%$ & 3 & $9.7 \%$ & 15 & $48.4 \%$ & 13 & $41.9 \%$ \\
Sexto & 0 & $0.0 \%$ & 1 & $3.6 \%$ & 12 & $42.9 \%$ & 15 & $53.6 \%$ \\
Sétimo & 2 & $7.4 \%$ & 5 & $18.5 \%$ & 15 & $55.6 \%$ & 5 & $18.5 \%$ \\
Oitavo & 0 & $0.0 \%$ & 5 & $23.8 \%$ & 9 & $42.9 \%$ & 7 & $33.3 \%$ \\
\hline Total & 2 & $1.2 \%$ & 22 & $13.7 \%$ & 77 & $47.8 \%$ & 60 & $37.3 \%$ \\
\hline
\end{tabular}

Legenda: ${ }^{*} p=0.0207$ Teste Qui-Quadrado Partição.

Fonte: SOARES AS, et al., 2020.

A opinião sobre a dificuldade de aprendizagem em neurologia devido à forma como está sendo ensinada, difere significativamente $\left({ }^{*} p=0.0090\right)$ entre os períodos. As maiores proporções foram encontradas na categoria "Moderado" com o sétimo, quinto e oitavo período (59.3\%, $58.1 \%$ e $52.4 \%)$, respectivamente.

\section{DISCUSSÃO}

$\mathrm{Na}$ instituição onde a pesquisa foi desenvolvida e aplicada, os discentes do curso de graduação em Medicina experimentam pela primeira vez o módulo de neurologia no $4^{\circ}$ (quarto) semestre do curso por meio de discussão em tutoria, aulas em laboratório morfofuncional e aulas teóricas ministradas por docentes sobre aspectos anatômicos e fisiológicos além de experiência semiológica em ambulatório de habilidades clínicas onde o conteúdo máximo exigido é a semiologia correta e a correlação com anátomo-fisiologia. $O$ aluno, posteriormente, revisita a neurologia no $7^{\circ}$ (sétimo) período do curso onde são abordadas as patologias neurológicas e a correlação da semiologia aprendida anteriormente com a condução e raciocínio clínico frente aos pacientes. Esse currículo pedagógico médico pode ser associado aos resultados encontrados na atual pesquisa.

Foi encontrado que os discentes do 4 ำríodo do curso, aqueles que visitam a especialidade pela primeira vez, relatam mais dificuldade de aprendizado, menor interesse em estudar e maior proporção de pouca confiança em atender emergências neurológicas e atendimento ambulatorial. Esse dado pode ser associado ao contato inicial com a especialidade que demanda além da preparação emocional de enfrentar uma especialidade comumente estigmatizada como "Difícil de aprender", também à grande carga de conteúdo que é ministrado de forma abstrata (estudo em livros textos e aulas teóricas) quando se deveria ser construída como uma metodologia pedagógica funcional tais como aulas em projeções, correlação com cadáver e até mesmo metodologias de ensino ministrada por professores que estimulam a construção do conhecimento, e não simplesmente o repasse de informações, sendo um resultado que encontra correlação na literatura médica ao avaliar que neurologia é a disciplina mais difícil, em que há menos confiança no exame físico e a de pior qualidade de aulas (SANTOS-LOBATO BL, et al., 2018).

Essa realidade é perigosa por ser um fator desencadeador da "neurofobia". A falta de aulas metodológicas pedagógicas que sejam acessíveis e desenvolvam a construção do conhecimento sem assustar o estudante de Medicina desde o primeiro contato com a neurologia, desenvolvem uma base de aversão à especialidade com relatos variando de "nunca conseguirei aprender pela enorme quantidade de assuntos" até "não tento entender por ser difícil" (MORINIGO D, et al., 2017) o que pode prejudicar o aprendizado de correlação do conhecimento anátomo-fisiológico com 0 patológico durante $07^{0}$ período e dificultar a conexão de informações da neurologia com outras áreas da medicina onde ocorrem doenças com repercussão neurológica. 
Dessa forma, além do prejuízo funcional para o estudante de Medicina que ao desistir de aprender sobre esta especialidade, permite o risco de futuramente realizar a semiologia incorreta, não solicitar exames imprescindíveis e negligenciar sinais e sintomas que podem ser precoces que, ao nível ambulatorial o médico encaminharia ao neurologista e, em nível de urgência, poderia evitar consequências desfavoráveis de pacientes sendo fatores primordiais que podem construir uma realidade como a de uma pesquisa em uma universidade privada que $48 \%$ dos estudantes já tiveram algum tipo de neurofobia durante o curso e até $50 \%$ relata que a forma de ensinar do professor pode ter influenciado no aprendizado da especialidade (RESTREPO J, et al., 2017). A forma de ensinar com a criação de barreiras para o aprendizado pode ser associado com metodologias arcaicas de ensino teórico com absorção do conhecimento sem discussão da praticidade dele ou visualização direta por meio das aulas práticas em ambulatórios, apoio multimídia ou discussões de casos clínicos.

Tendo em vista que o alvo da melhoria da neurofobia está nos estágios iniciais, durante o primeiro contato com a especialidade, a metodologia de aulas teóricas passivas pode dar espaço para discussão de casos clínicos com abordagens multimídias como a utilização de vídeos para demonstração de sintomas neurológicos e análise da neuroanatomia em cadáver, o qual tornaria as aulas mais práticas e palpáveis, diminuindo estatísticas encontradas pela atual pesquisa que maioria declarou que o conhecimento de neurologia varia de "pouco" e "moderado" e que 93,9\% dos estudantes classificaram o processo ensino aprendizagem em neurologia de difícil a muito difícil.

Contudo, essa não é uma realidade isolada. Em pesquisa realizada em 4 hospitais, foi encontrado que $54 \%$ dos estudantes apresentam neurofobia e $63,3 \%$ relatam a falta de conhecimento em neurologia básica como a causa mais importante (MORINIGO D, et al., 2017). Em outras diversas pesquisas conclui-se que a neurofobia é um traço forte e preponderante no aprendizado da especialidade, gerando dificuldades durante o curso, tais como uma desenvolvida na Arábia Saudita onde $84,4 \%$ dos estudantes possuem dificuldade em neurologia e até $42,7 \%$ relatam que possuem insuficiente conhecimento em neurociências (ABULABAN AA, et al., 2015) outra nos EUA que analisam que até $46 \%$ relatam que neurologia é a especialidade que tem mais dificuldade durante o curso e na Finlândia onde 35\% admitem que a "neurofobia" interferiu ou possa ter interferido na aprendizagem do conhecimento durante o estudo da especialidade (ANSAKORPI H, et al., 2017) demonstrando que os dados encontrados pela atual pesquisa não é uma realidade exclusivamente brasileira.

Dessa forma, os dados encontrados corroboram a necessidade de mais atividades práticas no início do contato com a neurologia. Essa afirmação encontra embasamento no resultado de que os estudantes do $7^{\circ}$ (sétimo) período que estão estudando neurologia pela segunda vez durante o curso e associando conhecimentos com patologias e do $8^{\circ}$ (oitavo) período o qual possui os conhecimentos mais frescos além de ter mais experiência no estudo e no atendimento clínico e, portanto participaram de mais aulas práticas, atendimentos e desenvolvimento de raciocínio clínico, possuíram resultados de avaliar menos a neurologia como uma especialidade difícil, possuírem mais interesse pela disciplina, apresentarem mais proporção de conhecimento "moderado", moderada confiança em atendimento de emergências neurológicas e atendimento ambulatorial, além de classificarem menos dificuldade no exame físico, menos dificuldade de aprendizagem pela imagem de ser difícil.

Esses dados apontam a tendência de queda da neurofobia sendo diretamente relacionado ao aumento de atividades práticas e metodologias pedagógicas por parte de professores/tutores e são corroborados pela literatura médica que analisam que a percepção negativa diminui com elaboração de aulas onde o estudante participa ativamente da construção do conhecimento (ABUSHOUK Al, et al., 2016; TAROLLI CG, et al., 2018) sendo esse um objetivo que visa aumentar a quantidade de discentes que veêm a neurologia com sentimentos e percepções positivas, diminuindo a neurofobia e aumentando a quantidade de egressos do curso de graduação que escolhem a neurologia como uma especialidade médica provável para sua vida (SOLORZANO GE, et al., 2015). Realidade encontrada em diversas instituições de ensino em saúde que iniciam o processo de transformação do ensino de assuntos da neurologia em forma de aulas teóricas para uma associação de aulas teóricas com aulas práticas para sedimentação do conhecimento e criação de conexões mentais dos assuntos com sua fisiopatologia, etiologia, quadro clínico, diagnóstico, classificação e tratamento/manejo. 


\section{CONCLUSÃO}

Logo, foi possível analisar a existência da neurofobia de acordo com a preparação e metodologia de ensino da especialidade durante a graduação médica, sendo a queda da neurofobia diretamente proporcional ao aumento de atividades práticas e aulas metodológicas pedagógicas que facilitam o entendimento e ensinamento da especialidade por parte do aluno. Além disso, são necessárias mais pesquisas na área da neurofobia e da pedagogia de educação médica a fim de que sejam diagnosticadas as particularidades da neurofobia em cada região e semestre do curso para a criação de medidas institucionais, pesquisadas e comprovadas para melhoria dessa realidade.

\section{REFERÊNCIAS}

1. ABULABAN AA, et al. Neurophobia among medical students. Neurosciences (Riyadh). 2015 Jan; 20(1): 37-40

2. ABUSHOUK AI, et al. Curing neurophobia in medical schools: evidence-based strategies Med Educ Online. 2016; 21: 10.3402/meo.v21.32476

3. ALBERT DA, et al. Structure of neuroscience clerkships in medical schools and matching in neuromedicine. Neurology 2015;85:172-6.

4. ANSAKORPI $\mathrm{H}$, et al. Medical students' experience of emotions and success in neurological studies - What do they tell us? BMC Medical Education, 2017; 17(1):68)

5. BRASIL. São Paulo State Regional Council of Medicine. Medical Demography in Brazil, 2015. São Paulo, Brazil: 2015.

6. FANTANEAUNU TA, et al. Neurophobia inception: a study of trainees' perceptions of neurology education. Canadian Journal o Neurological Sciences. 2014, 41(4): 421-429GELB DJ, Gunderson CH. The neurology clerkship core curriculum. Neurology. 2002;58:849-52.

7. FIESTAS F, et al. Improving mental and neurological health research in Latin America: a qualitative study. BMC Public Health. 2009;9:334.

8. JOZEFOWICZ RF. Neurophobia: The Fear of Neurology Among Medical Students. Arch Neurol. 1994;51(4):328-329.

9. MATTHIAS AT, et al. Neurophobia among medical students and non-specialist doctors in Sri Lanka. BMC Med Educ. 2013;13:164.

10. MORINIGO D, et al. Neurofobia en estudiantes de postgrado en Medicina Interna. Rev. virtual Soc. Parag. Med. Int., Asunción , 2017, 4(2): 42-48.

11. PRITHISHKUMAR IJ, HOLLA SJ. Early clinical exposure as a teaching learning tool to teach neuroanatomyfor first year occupational and physical therapy students - our preliminary experience. Indian Journal ofPhysiotherapy and Occupational Therapy - An International Journal. 2012;6(2):59-62.

12. SANTOS-LOBATO BL, et al. Neurophobia in Brazil: Detecting and Preventing a Global Issue. Rev. bras. educ. med., 2018, 42(1): 121-128.

13. SOLORZANO GE, et al. Neurophobia. A chronic disease of medical students. Neurology, 11 Jun 2015, 85(2):116117.

14. RESTREPO J, et al . Percepción de neurofobia en estudiantes de último año de Medicina en una universidad privada. Acta Neurol Colomb., 2017, 33(2): 63-67

15. TAROLLI CG, et al. Managing Neurophobia: How Can We Meet the Current and Future Needs of Our Students? Semin Neurol2018; 38(4):407-412 